\title{
INTERACTION OF EXCITONS BOUND TO $3 d$ TRANSITION METAL IONS WITH LATTICE VIBRATIONS IN II-VI SEMICONDUCTORS
}

\author{
V.I. SOKOLOV AND T.P. SURKOVA \\ Institute of Metal Physics, Academy of Sciences of the USSR, Ural Department, \\ Kovalevskaya 18, 620219 Sverdlovsk, GSP-170, USSR \\ (Received in final form November 22, 1990)
}

The analysis of an interaction of bound excitons with lattice vibrations for $\mathrm{ZnO}: \mathrm{Ni}$ and $\mathrm{ZnO}: \mathrm{Cu}$ is given on the basis of symmetry consideration.

PACS numbers: $78.20 . \mathrm{Wc}, 78.50 . \mathrm{Ge}$

A large body of experimental information on the interaction of lattice vibration with donor and acceptor excitons (DE and $\mathrm{AE}$ ) bound to isoelectronic impurities has recently been accumulated. This interaction is very strong. Apart from this, the observed peak position often does not correspond to the phonons from the centre of the Brillouin zone [1]. The analysis of experimental results is based on the fact that DE and AE substantially distort ionic oscillations near the impurity, owing to strong localization of the carrier captured by a non-Coulomb potential. This results in a phonon spectrum exhibiting normal modes of a cluster that consists of an impurity and some lattice ions surrounding it [2]. Excitons localized on an impurity interact with normal oscillations of a given claster.

When an impurity is introduced into the lattice, the motion of the ions that surround the impurity acquires the point symmetry of the impurity site, e.g. $T_{d}$ for crystals with zincblende structure. Combinations of atomic displacements near the impurity (normal modes) are described by irreducible representations $A_{1}$ (breathing mode), $E$ and $2 T_{2}$ of $T_{d}$ group. These are expressed in terms of irreducible representations of phonons of a perfect crystal. The contribution to normal modes originates from phonons from different Brillouin zone points, and the total number of phonons, that is, the projected density of phonon states, is calculated by a computer. Results of such computations for normal modes $A_{1}(\mathrm{ZnS}), E$ and $T_{2}$ (in $\mathrm{ZnS}, \mathrm{ZnSe}, \mathrm{ZnTe}$ ) are given in [2]. When the difference in force constants and/or ion masses is large, local or quasilocal lattice vibrations may appear. 
If the symmetry of the localized-carrier wavefunction is described by irreducible representations $\Gamma_{\alpha}$, the carrier will interact with the normal modes contained in symmetrized product $\left[\Gamma_{\alpha}^{2}\right]$. For $T_{d}$-structure crystals the wavefunction of an electron captured onto a simple isoelectronic impurity has a symmetry $\Gamma_{1}$, that of a hole the symmetry $\Gamma_{15}$ (disregarding spin). Consequently, the electron (and the $\mathrm{AE}$ ) will interact with the normal mode $A_{1}$, and the hole (and DE) with the normal modes $A_{1}, E_{1}, T_{2}$ [3]. When the exciton is localized on the $3 d$ impurity, one carrier contributes to the $d$ shell (changing the configuration $d^{n} \rightarrow d^{n+1}$ ) and becomes indistinguishable from the other $d$ electrons. Therefore one has to consider the interaction with lattice vibrations of the configurations $d^{n-1}$ and $d^{n+1}$ in the ground state for $\mathrm{DE}$ and $\mathrm{AE}$. For example, for $\mathrm{Ni}^{2+}\left(d^{8}\right.$ configuration) in the case of DE $\left[d^{7} e\right]$ capture, the ground state of the $d^{7}$ configuration is ${ }^{4} A_{2}$, in the case of $\mathrm{AE}\left[d^{9} h\right]$ capture, the ground state of the $d^{9}$ configuration is ${ }^{2} T_{2}$. With the neglect of spin, the DE will interact with the normal mode $A_{1}\left(\left[A_{2}^{2}\right] \rightarrow A_{1}\right)$, and the AE with $A_{1}, E$ and $T_{2}\left(\left[T_{2}^{2}\right] \rightarrow A_{1}+E+T_{2}\right)$. The table from [4] furnishes normal cluster oscillation modes with which $\mathrm{DE}$ and $\mathrm{AE}$ bound on $3 d$ impurities interact.

According to [2] the $A_{1}$ mode has only one peak in the region of optical vibrations (branch LO) but $E$ and $T_{2}$ modes have two peaks (LO and TO branches). This makes possible to distinquish $\mathrm{DE}$ and $\mathrm{AE}$ bound to $\mathrm{V}, \mathrm{Cr}, \mathrm{Ni}$ and $\mathrm{Cu}$. The predicted character of interaction of bound excitons with lattice vibrations is supported by examples of $\mathrm{DE}$ and $\mathrm{AE}$, observed for $\mathrm{ZnSe} \mathrm{Ni}, \mathrm{ZnS}: \mathrm{Ni}$ and $\mathrm{ZnTe}: \mathrm{O}$ [3, 4].

In the present paper we shall discuss experimental results concerning an interaction of excitons bound to $\mathrm{Ni}$ and $\mathrm{Cu}$ in $\mathrm{ZnO}$. The phonon spectrum consists of some optical branches $\Gamma_{1}, 2 \Gamma_{4}, \Gamma_{5}$ and $2 \Gamma_{6}$ (another symbols $A_{1}, 2 B_{1}, E_{1}$ and $2 E_{2}$ ). On projecting these branches onto the local vibration we see that the $\Gamma_{1}$ and $\Gamma_{4}$ phonons make a contribution to local $A_{1}$ mode, and the $\Gamma_{5}$ phonons contribute to the local mode $E$. Figure 1 shows the spectral dependence of electroabsorption harmonic $\alpha_{2}$ for $\mathrm{ZnO}: \mathrm{Ni}$ and the spectra of luminescence and excitation of luminescence for $\mathrm{ZnO}: \mathrm{Cu}$ [5]. It should be noted some simularity in these two cases: the peaks 1,2 and 3 of $\alpha_{2}$ spectrum and the peaks $\beta$ and $\gamma$ of the excitation luminescence spectrum, broad structures 4 and 5 of the $\alpha_{2}$ spectrum and the broad peaks for the luminescence spectrum. Both the more strong peak 6 for the $\alpha_{2}$ spectrum and the corresponding peak for the luminescence spectrum are determined by optical phonons $\Gamma_{6}\left(A_{1 L}\right)$. The wide peaks 4 and 5 for the $\alpha_{2}$ spectrum are interpreted as $\Gamma_{4}\left(B_{1}\right)$ replicas. $\Gamma_{4}$ phonons are forbidden by symmetry for an observation in optical absorption and Raman scattering spectra. In our case these phonons make a contribution to the local mode $A_{1}\left(\left[\Gamma_{4}^{2}\right] \rightarrow A_{1}\right)$ and therefore they are observed in electroabsorption and luminescence spectra.

It is important to note that TO phonons $\Gamma_{5}\left(E_{1}\right)$ in the spectrum of $\mathrm{ZnO}: \mathrm{Ni}$ and in the luminescence and excitation luminescence spectra of $\mathrm{ZnO}: \mathrm{Cu}$ are not displayed as a very strong peak. This can be understood for $\mathrm{AE}\left[d^{10} h\right]$, because of its interaction with $A_{1}$ mode only. The resemblance of the vibrational replicas structure of $\mathrm{ZPL}$ for $\mathrm{ZnO}: \mathrm{Cu}$ and $\mathrm{ZnO}: \mathrm{Ni}$ allows to make a conclusion that in the case of $\mathrm{ZnO}: \mathrm{Ni} \mathrm{DE}\left[d^{7} e\right]$ is observed, which interacts only with $A_{1}$ mode. For 


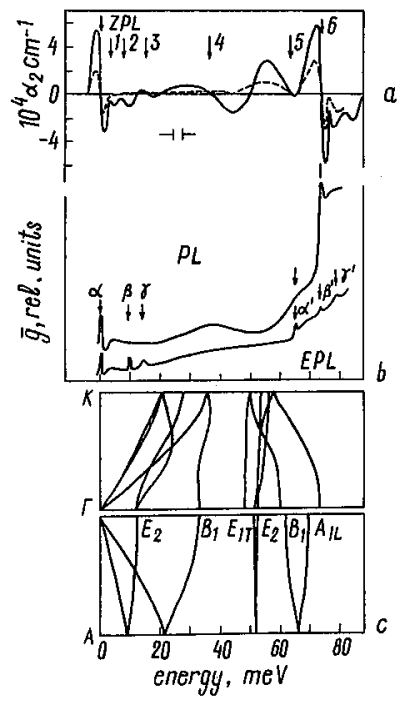

Fig.1. Spectral dependence of the EA second-Harmonic amplitude $\alpha_{2}$ for ZnO:Ni (a), the photoluminescence (PL) and excitation photoluminescence (EPL) for $\mathrm{ZnO}: \mathrm{Cu}$ (b) [5], and dispersion curves of normal lattice vibration modes in $\mathrm{ZnO}$ (c) [6]. The position of $\mathrm{ZPL}$ at the $2.152 \mathrm{eV}$ for $\mathrm{DE}$ in $\mathrm{ZnO}: \mathrm{Ni}$ and $2.864 \mathrm{eV}$ for $\mathrm{AE}$ in $\mathrm{ZnO}: \mathrm{Cu}$ are given as zero at energy scales for $a, b$.

a quantitative discussion it is necessery to perfom a calculation of the projected density-of-states for $\mathrm{ZnO}: \mathrm{Cu}$ and $\mathrm{ZnO}: \mathrm{Ni}$.

\section{References}

[1] V.I. Sokolov, K.A. Kikoin, Soviet Scientific Reviews A, Physics 12, 147 (1989).

[2] A.L. Natadze, A.L. Ryskin, L.G. Verhter, in The Dynamical Jahn-Teller Effect in Localized Systems, ed. Ju.E. Perlin and M. Wagner, Elsevier Science Publishers, 1984, p.394.

[3] J.J. Hopfield, D.G. Thomas, R.T. Lynch, Phys. Rev. Lett. 17, 312 (1966).

[4] V.I. Sokolov,T.P. Surkova, Fiz. Tverd. Tela 29, 2938 (1987).

[5] R. Dingle, Phys. Rev. Lett. 23, 579 (1969).

[6] N. Vagelatos, D. Weke, J.S. King, J. Chem. Phys. 60, 3613 (1974). 